\title{
Von der Objektifizierung zur Quantifizierung ${ }^{1}$ Wandlungen des invektiven Blicks
}

Jens Schröter

\section{Abstract}

Wenn man nach dem invektiven Blick (invective gaze) im digitalen Bild fragt und das digitale Bild in einer "kapitalistisch infizierten Bilderlogik " ${ }^{2}$ verortet, steht man vor der theoretischen Herausforderung, den Blick, das Digitale und den Kapitalismus zusammenzudenken. Alle drei Begriffe sind groß skaliert und theoretisch umstritten - dennoch will der vorliegende Aufsatz versuchen, ihre theoretische Relation - ausgehend von der Theorie des Blicks - zumindest zu skizzieren, um sich damit der Frage nach einem »invektiven " (herabsetzenden) Blick im heutigen digitalen Bild (oder jedenfalls: bestimmten Formen dieses Bildes) anzunähern.

Beginnt man also mit dem Blick, so gibt es mindestens zwei signifikante theoretische Auseinandersetzungen - nämlich jene von Jean-Paul Sartre und die von Jacques Lacan. Es ist hier kaum möglich, die umfangreiche und weit verzweigte Diskussion um diese, teilweise enigmatischen, Ansätze wiederzugeben. Entscheidend ist nur, dass beide betonen, dass es zur wesentlichen Grundverfassung des Subjekts gehört, sichtbar zu sein.

Sartre schreibt in seinem 1943 erstmals erschienenen Buch Das Sein und das Nichts:

Nehmen wir an, ich sei aus Eifersucht, aus Neugier, aus Verdorbenheit so weit gekommen, mein Ohr an eine Tür zu legen, durch ein Schlüsselloch zu gucken. [...] Jetzt habe ich Schritte im Flur gehört: man sieht mich. Was soll das heißen? [...] [D]ie Scham oder der Stolz enthüllen mir den Blick des Andern und mich selbst am Ziel dieses Blicks, sie lassen mich die Situation eines Erblickten erleben, nicht erkennen. Die Scham aber ist [...] Scham über sich, sie ist Anerkennung dessen, 
daß ich wirklich dieses Objekt bin, das der Andere anblickt und beurteilt. (Sartre 1991: 467-471)

Sartre beschreibt also eine Situation, in der ein selbst zunächst unbemerktes Subjekt heimlich andere beobachtet - also souverän die Sichtbarkeit beherrscht. Doch dann wird es ertappt - interessanterweise kündigt sich das eigene Gesehenwerden in Geräuschen (die Schritte) an. In diesem Moment wird sich das Subjekt bewusst, dass es sichtbar ist und dass es durch diese Sichtbarkeit von dem beobachtenden Anderen verurteilt werden kann. Man empfindet Scham. Man fühlt sich herabgesetzt durch den Blick des Anderen.

Diese Beschreibung des Blicks hat um 1964 Jacques Lacan aufgegriffen und radikalisiert. ${ }^{3}$ Ohne hier eine tiefere Diskussion über Lacans Theorie des Blicks durchführen $\mathrm{zu}$ können und $\mathrm{zu}$ wollen (siehe dazu u.a. Blümle/von der Heiden 2005), kann man festhalten, dass die Sichtbarkeit zu einem konstitutiven Merkmal von Subjektivität erklärt wird: »[I]ch sehe nur von einem Punkt aus, bin aber in meiner Existenz von überall her erblickt (Lacan 1987: 78). Wir sind »angeschaute Wesen " (ebd.: 81). "Der Blick, um den es hier geht, ist also in der Tat Gegenwart des andern als solchen « (ebd.: 91). ${ }^{4}$ "Das Bild ist sicher in meinem Auge. Aber ich, ich bin im Tableau« (ebd.: 102). In der berühmt gewordenen Stelle, wenige Seiten später spitzt Lacan folgendermaßen zu:

Von Grund aus bestimmt mich im Sichtbaren der Blick, der im Außen ist. Durch den Blick trete ich ins Licht, und über den Blick werde ich der Wirkung desselben teilhaftig. Daraus geht hervor, daß der Blick ein Instrument darstellt, mit dessen Hilfe das Licht sich verkörpert, und aus diesem Grund auch werde ich [...] photographiert (ebd.: 113)

Schließlich heißt es: »Es ist, betrachtet man die Universalität der Funktion des bösen Blicks, überraschend, daß es nirgends auch nur die Spur eines guten Blicks, eines Auges, das Segen bringt, gibt« (ebd.: 122). Es seien drei Punkte herausgehoben, die besonders die Verbindung des Blicks mit Bildtechnologien betonen:

1. Lacan unterscheidet, visualisiert durch drei berühmte Diagramme (ebd.: 97, 112), ${ }^{5}$ zwei Formen oder besser Konzeptualisierungen des Blicks: jenen, in dem das

3 Mit der Zwischenstufe Merleau-Ponty (1986), worauf hier nicht eingegangen werden kann. Vgl. auch Därmann 2000.

4 Condek (1997: 184) weist daraufhin, dass »Lacan selbst kein einziges Mal vom >Blick des Anderen<spricht«. Dennoch scheint mir Lacans Formulierung eindeutig auf intersubjektive Sichtbarkeit zu zielen.

5 Wegen des klaren Kontrastes thematisiere ich nur die ersten beiden Diagramme und erlaube mir, das dritte zu überspringen. Überhaupt dürfte meine Lacan-Lektüre ungenau und fehlerhaft sein - ich experimentiere ein bisschen mit dem Text und bitte dafür um Nachsicht. 
Abb. 1: Die drei Diagramme des Blicks bei Lacan (1987: 97, 112).

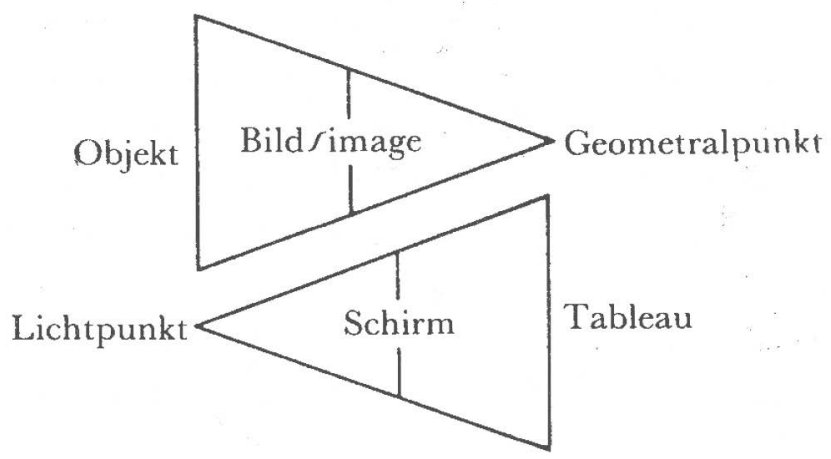

Subjekt - ähnlich wie Sartres Schlüsselloch-Seher - souverän eine Szene überblickt, Träger des Blicks ist. Im Englischen kann man dies als look bezeichnen. Lacan bringt dieses Sehen in Verbindung mit der Zentralperspektive. Das erste Schema sieht genauso aus wie entsprechende Darstellungen der zentralperspektivischen Projektion und Lacan nennt es "geometral« (ebd.: 91). Das Sichtbare ist auf einen Augenpunkt hin ausgerichtet. Diese Vorstellung des Sehens ist aber in gewisser Weise illusorisch - ähnlich wie bei Sartre wird die Souveränität des Sehenden durch ein »eigentlicheres« (ebd.: 98) Sehen überrumpelt.

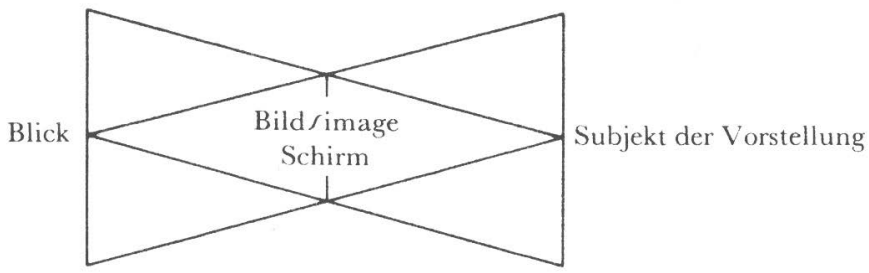

Denn bei der zweiten Form des Blicks - im Englischen the gaze - ist das Subjekt, ähnlich wie in dem Moment, wo man bei Sartre ertappt wird, Objekt des Blicks. Das zweite Schema, das merkwürdig an die Situation des Kinos erinnert, geht von einem »Lichtpunkt « der Blick aus und das Subjekt ist »Tableau« (ebd.). Das Subjekt ist Sichtbares und radikaler als bei Sartre: wird potenziell immer gesehen. Das Angesehenwerden gehört hier zur Struktur der Subjektivität. Jede/r will 
'gut aussehen - in unserer patriarchalen Kultur ist insbesondere der weibliche Körper Objekt fortlaufender Visualisierung und visueller Korrektur (und selbst in der Verweigerung spiegelt sich noch dieser Imperativ). ${ }^{6}$ Zwischen den Blick und das Subjekt schiebt sich ein »Schirm « - wie man vermuten kann, ist der Schirm das »image repertoire« (Silverman 1996: 134f.; siehe auch Bryson 1988: 91f.), welches menschlichen Subjekten als Vor-Bild ihrer eigenen Visualisierung dient (z.B. Schönheitsideale, Mode, Starkörper etc.). ${ }^{7}$ Der Schirm ist aber auch Schutz - man könnte sagen: Indem wir uns sgefällig und kon-form geben, schützen wir uns vor der Scham und dem (invektiven) Blick.

2. Lacan spricht von »Instrument« und davon, dass das Subjekt sozusagen strukturell »photo-graphiert« sei. Diese Formulierung, das herangezogene Dispositiv der Zentralperspektive und die immer wieder erwähnte Malerei (im Seminar XI: Goya, Rouault, Holbein, Caravaggio, Cezanne ${ }^{8}$ ) legen nahe, dass Bildtechnologien in einer noch näher zu bestimmenden Weise für den Blick - die Sichtbarkeit des Subjekts - eine konstitutive Rolle spielen. Man kann schon hier mutmaßen, dass sie mit dem, ebenfalls nach bestimmten Verfahren von Bildtechnologien benannten Schirm in enger Verbindung stehen. Die oben zitierte Bestimmung des Schirms als "Ort der Vermittlung« (Lacan 1987: 114), als dem Reservoir massenmedialer Bilder, nach denen wir uns formen, deutet darauf hin.

3. Der Blick scheint gegenüber dem Subjekt übermächtig, er wird mit dem »bösen Blick« assoziiert, es heißt gar, dass es »jenseits des Scheins zwar kein Ding an sich, aber den Blick « (ebd.: 110) gäbe. Der Blick scheint das Subjekt zu beherrschen, in Scham zu versetzen, überhaupt erst zu subjektivieren, er ist die »Kehrseite des Bewußtseins« (ebd.: 90). Auch wenn Lacan einräumt, dass man mit dem »Schirm« "spielen« kann (ebd.: 114), hat der Blick doch eine "paranoid coloration« (Bryson 1988: 104).

Daher hat eine wichtige feministische und queere Theoriebildung an verschiedene Aspekte der Theorie Lacans anschließen können - nicht ohne den ahistorischen und phallogozentrischen Charakter des Ansatzes zu befragen. Vgl. die Darstellung bei Jay 1993: 542ff.

7 Offenkundig steht der Schirm in einem Verhältnis zum Spiegel und zum Imaginären, was hier nicht diskutiert werden kann. Siehe dazu Jay 1993: 329-370.

8 Vgl. Lacan 1987: 107: »Im Bild manifestiert sich mit Sicherheit immer ein Blickhaftes« - wie dies mit abstrakten Bildern zusammengeht oder solchen, die auf nicht-optischen, z.B. parallelperspektivischen, Konstruktionsverfahren basieren, ist eine schwierige Frage, die hier nicht erörtert werden kann. Vgl. Beil/Schröter 2011. 


\section{II.}

Diese drei Punkte seien nun weiter diskutiert.

1. Wenn der Blick und die Sichtbarkeit des Subjekts so fundamental für seine Konstitution sind, ist "the gaze [...] as old as sociality itself (Silverman 1996: 132) und mithin »transhistorical« (ebd.: 133) - und überdies eurozentrisch: Immerhin war schon 1952 Frantz Fanons wichtige Studie Peau noire, masques blancs erschienen. ${ }^{9}$ Doch Silverman führt die Historizität über den Schirm wieder ein (ebd.: 134) - das Bildrepertoire, nach welchem wir uns modellieren, ist wandelbar. Schönheitsideale können sich ändern. ${ }^{10}$ Es gibt also mindestens eine Spannung zwischen - wenn man so will - anthropologischen und historischen Bestimmungen des Sichtbar-Seins.

2. Silverman geht nun aber noch weiter. Sie fragt sich, unter Bezugnahme auf Lacans mysteriöse Formulierung, dass das Subjekt "photo-graphiert « werde, ob die Bildtechnologien nicht selbst unterschiedliche historische Formen seien, in denen das Regime des Blicks sich »verkörpert «. ${ }^{11}$ "At least since the Renaissance, optical devices have played a central role in determining how the gaze is apprehended, and such devices cannot simply be reduced to a set of images. (Ebd.: 136). Unter Rückgriff auf etwa Barthes' Beschreibungen, wie man im Moment des (bewussten) Fotografiertwerdens erstarrt, ${ }^{12}$ heißt es:

Fanon war von Sartre und auch Merleau-Ponty beeinflusst, selber Psychoanalytiker und benutzt schon im Titel den Begriff der Maske, der bei Lacan (1987: 90 und 114) auftaucht. Im Vorwort zur Ausgabe von 1986 schreibt Homi K. Bhabha (in: Fanon 2008: xxviii): [T]o exist is to be called into being in relation to an Otherness, its look or locus « und (xxix): »For Fanon, like Lacan, the primary moments of such a repetition of the self lie in the desire of the look." Auch hier spielt der Blick eine zentrale Rolle - so beginnt Kapitel 5, »The Fact of Blackness« (82ff.) u.a. mit der Zitierung des rassistischen Ausrufs und des damit aufgerufenen invektiven Blicks: »Look, a Negro!«. Aber die rassistische Strukturierung des Schirms wird bei Lacan, zumindest im Seminar XI, nicht erwähnt. Der transhistorische gaze scheint weiß zu sein. Auch wäre eine Frage, wie in einer Theorie des Blicks eigentlich blinde Menschen konzeptualisiert werden. Blindheit taucht bei Lacan (1987: 98f.) auf, aber nur negativ darin, dass die »geometrale«Auffassung des Sehens eine zentralperspektivische Projektion beschreibt, die auch von Blinden mit Fäden konstruiert werden kann. Das stimmt zwar, aber wenn so das »Eigentliche des Sehens « (ebd.: 98) verfehlt wird, welchen >uneigentlichen «Status haben dann blinde Subjekte? Vgl. Lacan 1986: 13: »Noch der Blinde ist [...] Subjekt, weil er sich als Objekt des Blickes weiß.»

10 Versuche der Historisierung Lacans wurden kritisch als eine Annäherung an Foucault beschrieben, vgl. Copjec 1994.

11 Vgl. Lacan 1987: 90: »Wenn also der Blick die Kehrseite des Bewußtseins ist, wie wäre er dann bildlich zu denken? Wir drücken uns hier nicht falsch aus, denn es ist möglich den Blick zu verkörpern.«

12 Vgl. Barthes 1989: 18f.: „Sobald ich nun das Objektiv auf mich gerichtet fühle, ist alles anders: ich nehme eine sposierende Haltung ein, schaffe mir auf der Stelle einen anderen Körper, 
[T] he camera has been installed ever since the early nineteenth century as the primary trope through which the Western subject apprehends the gaze. [...] [W] hen a real camera is trained upon us, we feel ourselves subjectively constituted, as if the resulting photograph could somehow determine $>$ who $<$ we are. (Ebd.: 135) ${ }^{13}$

Das stimmt - und nicht nur hinsichtlich des Erstarrens vor der Kamera, des Posierens (vgl. Owens 2003), sondern auch bezüglich solcher Phänomene wie Passfotos, die im Kontext von Identitäts-Dokumenten Subjektivität, die Verknüpfung von Aussehen und Namen und damit die politische und ökonomische Performativität des Körpers regulieren (vgl. Schröter 2016).

Ruth Iskin (1997) hat, etwa zeitgleich mit, aber ohne Bezug auf Kaja Silverman, an diesen Diskurs angeschlossen: "How ha[s] the radical increased role of imaging technologies affected our notions of human subjectivity? (Iskin 1997: 43). Sie betont ebenfalls die subjektkonstitutive Rolle des durch den Schirm (und seine medialen Realisierungen) vermittelten Blicks (ebd.: 48, 52). Damit taucht die Frage auf, ob andere Technologien auch andere historisch spezifische Formen des Blicks hervorbringen: "Lacan alludes to the fact that formations of subjectivities are deeply affected by specificities of the signification system, its apparatuses and media and, we might add, their historically specific articulations and roles in the social arena.« (Ebd.: 58)

Das sind wichtige Hinweise, allerdings führt Iskin nicht im Detail aus, welche unterschiedlichen Effekte z.B. "photography, film and TV« (ebd.: 58) oder »cyberspace« (ebd.: 59) genau haben könnten. Sie bezeichnet etwa das Display von Computern als "post-industrial >mirrors of images that often continue to refer to photographic representation, whether they are synthetically generated or manipulated with the computer, or are transmitted from a photographically produced source«. (Ebd.: 53) Hier werden sehr verschiedene Bildtypen genannt, die mit Computern erzeugt werden können - und es scheint dabei wichtig zu sein, dass sie auf die eine oder andere Weise auf Fotografie Bezug nehmen. Es stimmt, dass viele (aber keineswegs alle) Formen der Computergrafik auf die Fotografie Bezug nehmen, bis hin zur Modellierung einer >virtuellen Kamera (vgl. Schröter 2003; zur komplexen Begrifflichkeit des digitalen Bildes siehe auch Schröter 2004). Und es ist richtig,

verwandle mich bereits im voraus zum Bild. Diese Umformung ist eine aktive: ich spüre, daß die PHOTOCRAPHIE meinen Körper erschafft oder ihn abtötet, ganz nach ihrem Belieben.« Zu Barthes und Lacan vgl. Iversen 2002.

13 Es gab und gibt eine Reihe von medientechnologischen Metaphern für Bewusstsein, Unbewusstes, Cedächtnis etc., die keineswegs immer optischen Medien nachempfunden sind (das gilt wohl auch für den oft diskutierten Wunderblock bei Freud, der aber immerhin noch gesehen, jedoch auch beschrieben werden muss), aber das kann hier nicht weiter ausgeführt werden. 
dass alle Bildmedien Licht aufzeichnen und/oder generieren und/oder reflektieren und/oder abstrahlen - aber ein so allgemeiner Bezug auf das Licht würde die anvisierte medienhistorische Ausdifferenzierung des Blicks ja eher undeutlicher machen. Auch Silverman (1996: 195) nennt "photography, cinema and video« und sie verbindet diese Technologien darüber, dass sie alle dem Dispositiv der Kamera folgen. Das hieße aber, dass etwa ihre unterschiedlichen Modalitäten der Aufzeichnung (still/bewegt, fotochemisch/elektronisch, analog/digital) keine Rolle spielen.

Und warum auch nicht: Macht es für das Angeblicktwerden, das Erstarren, die Pose z.B. einen Unterschied, ob die Kamera analog oder digital aufzeichnet? Wohl eher nicht. Könnte es einen spezifischen digitalen Blick geben? ${ }^{14}$ Immerhin bemerkt Silverman in ihrer Lektüre von Harun Farockis Film Bilder der Welt und die Inschrift des Krieges (1988): »The camera/gaze thus emerges within Bilder as an apparatus for the production of quantified and quantifiable images." (Ebd.: 143) Gibt es mithin jenseits des objektifizierenden Blicks, wie ihn drastisch Sartre beschrieben hat, auch einen quantifizierenden Blick - auf den schon, wenn auch in einem ganz anderen Kontext und mit anderen Begrifflichkeiten, Baxandall (1988) hingewiesen hat? Wären digitale Aufzeichnungen der Quantifizierung und mithin Analyse nicht zugänglicher (siehe Gesichtserkennung)? Könnte also der Blick nicht - im Verbund mit Foucault'scher Überwachung - noch tiefer dringen, noch stärker subjektivieren, unterwerfen? Und wie würde diese Quantifizierung des Blicks mit der Frage nach der Ökonomie des Blicks zusammenhängen, denn eine Ökonomie quantifiziert notwendigerweise ${ }^{15}$ Und wie hängt dies wiederum mit der Ökonomie jener Bildtechnologien und ihrer Formen wie Inhalte zusammen, die zumindest einen Teil des Schirms bilden?

Dass die Ökonomie auch in den eher abstrakten Überlegungen Lacans keineswegs abwesend ist, zeigt die vielzitierte Anekdote zur Sardinenbüchse. Der junge Lacan ist auf einem Fischerboot und sieht auf den Wellen eine in der Sonne glitzernde Sardinenbüchse: »Da schwamm sie also in der Sonne, als Zeuge der Konservenindustrie, die wir ja beliefern sollten. Spiegelte in der Sonne. Und Petit-Jean

14 Wenn man, wie z.B. Kittler (1986) jedoch das Digitale (vermittelt über die Schreibmaschine) nahe an die diskrete symbolische Ordnung nach Lacan rückt, den Blick (vermittelt über das Kino) aber an das Imaginäre, könnte es eigentlich keinen digitalen Blick geben. Jedoch könnte es entweder sein, dass die Zuordnung des Blicks - dem Lacan (1987: 122) immerhin eine diskretisierende »Separationsgewalt « zuspricht - zum Imaginären zu kurz greift, oder, dass die Zuordnung medialer Dispositive zu bestimmten Registern bei Lacan selbst historisch veränderlich ist (wenn sie überhaupt Sinn ergibt).

15 Eine kapitalistische Ökonomie (wenn man sich darüber verständigen kann, was Kapitalismus bedeutet) weist allen Objekten Tauschwert, also letztlich einen quantifizierbaren Preis, zu. Aber auch eine nicht-kapitalistische Ökonomie müsste mit Quantitäten rechnen, z.B. wenn es darum geht, zu bestimmen, wie viel Mehl zu welcher Zeit an welchem Ort sein müsste, damit (zumindest ungefähr) wie viele Brötchen gebacken werden können. 
meinte: - Siehst Du die Büchse? Siehst Du sie? Sie, sie sieht dich nicht!« (Lacan 1987: 101). Diese Szene dreht sich also um eine industriell hergestellte Büchse, die den jungen Lacan »anblickt, angeht « als er mit diesen »Leuten, die so schwer für ihre Existenz $\mathrm{zu}$ schuften hatten « (ebd.: 102), unterwegs ist. Arbeit und Industrie sind anwesend in dieser Szene. Es geht um materielle Güter wie Fische und Konservendosen (und nicht etwa um Informations- oder Kommunikationsarbeit), also um schwere körperliche Arbeit, mutmaßlich ausgebeutet von einer »Großindustrie« (ebd.: 101). Ein Problem, das den jungen Lacan angeht. ${ }^{16}$

3. Wenn der Blick bei Sartre doch per se, sofern er das Subjekt in Scham versetzt, herabwürdigend und bei Lacan eo ipso böse ist, ${ }^{17}$ wie hebt sich davon ein invektiver Blick $\mathrm{ab}$ ? Wäre nach Sartre und Lacan nicht jeder Blick invektiv, mindestens insofern er objektifizierend ist? Allerdings: Sartre (1991: 471) schreibt auch vom »Stolz«, den der Blick des Anderen auslösen kann, und Lacan spricht - hier zeigt sich erneut das oben schon angemerkte Gendering des Blicks als männlich - von der »Befriedigung einer Frau, die sich betrachtet weiß« (Lacan 1987: 81), und weiterhin vom "Blick des Liebhabers« (ebd.: 107). Welchen Sinn hätte auch das Spiel mit dem Schirm (ebd.: 114), z.B. in Formen der Selbst-Inszenierung, ${ }^{18}$ wenn nicht den, von anderen anerkannt und gewürdigt zu werden, also nicht-invektive Blicke auf sich zu ziehen? Ist der Flirt nicht genau diese Operation? Dass der Schirm zu Zwecken der »intimidation « (Silverman 1996: 134) genutzt werden kann, impliziert zwingend, dass er auch anders genutzt werden kann. Also kommt man nun zu der Frage, welches Spiel mit dem Schirm im Rahmen welcher medialen Situationen invektive oder nicht-invektive Blicke hervorbringt und welche Subjektivitäten dies (zumindest potenziell) erzeugen könnte. ${ }^{19}$

III.

Wenn man annimmt, dass der Blick ahistorisch ist und mithin gar nicht soziotechnisch ausdifferenziert werden kann, erübrigt sich die weitere Diskussion.

16 Zu Marx und Lacan siehe Tomšič 2015.

17 Vgl. Lacan 1987: 125, der den bösen Blick erneut in (moment-)fotografischen Metaphern beschreibt: »Der böse Blick, ist das fascinum, was durch seine Wirkung die Bewegung stocken läßt [...].«

18 Silverman (1996: 134) spricht daher auch von »travesty«, die der Schirm erlaubt (vgl. auch 195227 zu Analysen von Cindy Shermans selbstinszenatorischen Fotos, die den Schirm, der »die Frau« definiert, kritisch verfremdet). Hier ist die Nähe zur Thematik des drag naheliegend, vgl. Butler 1999 und neuerdings Görling 2017.

19 Grundsätzlich drängt sich die Frage auf, wie ein Subjekt, das in gewisser Weise durch den Blick gebildet wird, wiederum durch Blicke herabgesetzt werden kann. 
Wenn man jedoch annimmt, eine sozio-technische und historische Ausdifferenzierung sei möglich, und die kurz diskutierten Positionen von Silverman und Iskin deuten das an, dann eröffnet sich wahrscheinlich ein sehr weites und differenziertes Feld, das hier nicht en détail dargestellt werden kann.

Was sind z.B. Selfies aus der Sicht der hier diskutierten Theorie des Blicks? Und was nützt es, sie mit dieser Theorie (sofern es eine ist) zu beschreiben? Selfies sind ein Blick von Außen auf mich und für die Anderen bestimmt - aber von mir selbst durchgeführt und selbst inszeniert. Ich werde »photo-graphiert«, aber willentlich von mir selbst. Ist das nun ein look, insofern ich mich selbst souverän fotografiere (und dann manchmal noch, mehr Lacan geht ja gar nicht, verwirrenderweise, mithilfe eines Spiegels)? Oder ist es schon the gaze, insofern ich mich als mich selbst fotografierende/ $r$ unter das Regime der anderen als solcher stelle, denn das Bild ist ja gerade nicht für meinen Blick gemacht? Ein Selfie will einen nicht-invektiven Blick erheischen, kann aber dekontextualisiert invektiv werden. Ein Bild, das ich von mir selbst mache und von dem ich glaube, dass es mich optimal präsentiert, kann plötzlich, in sozialen Netzwerken, zu Scham und Herabsetzung führen. Kann man also Instagram-Filter als Spiel mit dem Schirm beschreiben? Ein Spiel, das der perfekten Selbstinszenierung dienen soll, die wiederum, ökonomisch, Teil einer Durchsetzungsstrategie am Aufmerksamkeits-Markt ist - und doch jederzeit wieder ins Lächerliche und Peinliche umschlagen kann ${ }^{20}$ Hier deutet sich ein erster Hinweis darauf an, was man als eine medienhistorisch eigene Form des Blicks in der Gegenwart sehen könnte. Diese und eine weitere Form des digital-invektiven Blicks seien im Folgenden diskutiert.

1. Es wäre zunächst die Zirkulation der eigenen Bilder zu nennen. Es gibt nicht mehr eine private Bildersphäre, z.B. von Familienfotos eingeklebt in Alben, der eine massenmediale Bildersphäre in Kino und TV etwa gegenübersteht, durch die Körperbilder von Models etc. kommuniziert und imaginär in Subjekte eingeprägt werden. So hat Lacan interessanterweise in Zusammenhang mit den »Massenmedien« bemerkt, dass ein »Überhandnehmen« des Blicks zu konstatieren sei: »Tatsächlich ist durch die Unzahl der Spektakel und Phantasmagorien nicht so sehr unser Sehen angesprochen als vielmehr der Blick.« (Lacan 1987: 288f.). Jede/r kann sich im Prinzip selbst abbilden und die Bilder, in digitalen Datennetzen, global zirkulieren lassen - was dennoch heißt, dass diese eigenen Bilder dem hegemonialen Schirm folgen. Das Konkurrenzprinzip bedeutet dann nicht, ich bin schöner, attraktiver,

$20 \mathrm{Vgl}$. https://www.for-me-online.de/beauty/haut-und-koerperpflege/instagram-filter [letzter Zugriff 14.7.2021]: »Ruckzuck lassen sich Fotos optimieren und uns prompt noch besser aussehen. Damit Ihr nächster Instagram-Post die Herzen der Follower:innen im Sturm erobert, stellen wir Ihnen jetzt unsere vier liebsten Instagram-Filter vor! « Offenkundig geht es um das Begehren, darum, Herzen im Sturm zu erobern. 
fröhlicher, erfolgreicher als Du, sondern ich bin den schönen, attraktiven, fröhlichen, erfolgreichen Models und Stars ähnlicher als Du. Die digitale Zirkulation des Selfies führt zur Konvergenz von Markt und Schirm. Die empirischen Studien von großen Mengen von Selfies, die etwa Lev Manovich dankenswerterweise durchgeführt hat (vgl. Tifentale/Manovich 2015), führen zu der erwartbaren Erkenntnis, dass diese Bilder normalverteilt im Wesentlichen gleich aussehen. Alle folgen dem Schirm (insbesondere die Frauen, vgl. ebd.: 115). Nur ist dies nun in der Selbstinszenierung und ihrer zirkulativen Präsenz sichtbar. Gerade das verführt zum Willen zur Abweichung, zur travesty.

Doch das ist immer problematisch. Billie Eilish ist das aktuelle Beispiel. Die junge Sängerin verhüllt sich in zu weite Kleidungen und Brillen etc., um nicht $\mathrm{zu}$ viel von ihrer Körperlichkeit preiszugeben, eben um gerade kein hegemoniales Bild zu sein. Geschieht das dann doch, weil irgendwelche Bikini-Fotos aus dem Urlaub eben zirkulativ geleakt werden, ist das Geschrei riesig (vgl. Sycha 2020). Plötzlich erscheint die depressive und verhüllte Billie als sexuell attraktive junge Frau (wen überrascht das?). Das, "was ich erblicke [ist], nie das, was ich sehen will (Lacan 1987: 109; Hervorhebung im Original). Wieder ist die scheinbare Opposition nur eine Bestätigung des Schirms und die wilden, hasserfüllten Reaktionen lassen sich gar nicht anders erklären, als Effekt der traumatischen Ent-täuschung, dass es »jenseits des Scheins [Eilishs Selbstinzenierung, JS] zwar kein Ding an sich, aber den [männlichen, ${ }^{21} \mathrm{JS}$ ] Blick« (ebd.: 110) gibt. ${ }^{22}$ Hier ist der invektive Blick einer, der aus der unerträglichen Enttäuschung geboren wird, dass es am Ende eben doch nur die kapitalistische Erscheinungsindustrie ist, die den Schirm produziert. Ja, dass es einfach kein Jenseits des Schirms gibt - bestenfalls ein Spiel mit ihm (so scheint es jedenfalls). Das ist für die eigene, vermeintlich oppositionelle Subjektivität - und so wird Billie Eilish zumindest verkauft - ein schwerer Schlag. Das geht einen an. Es richtet sich ein invektiver Blick auf Eilish, eben weil die Zirkulation einer, unter anderen medialen Bedingungen, unsichtbaren Privatheit, das gerade gegen den herrschenden Blick aufgebaute image unterbricht. So etwas findet man dann »weniger komisch « (ebd.: 101). Es ist wohl naheliegend, dass das Netz für die Formen der potenziell invektiven Blicke eigene Psycho-Formate des Vergessens und der Flucht vor der Scham entwickelt hat - wie etwa in flüchtigen Bildern bei Snapchat (vgl. Schröter 2019). ${ }^{23}$ Vielleicht kann der invektive Blick ja auch wieder verschwinden. Das ist jedenfalls die Hoffnung.

21 Siehe Jay 1993: 533, der vom »screen of male representations « spricht.

22 Vgl. auch Lacan 1987: 118f. zu der Geschichte von Zeuxis und Parrhasios: „Dagegen zeigt das Beispiel von Parrhasios, wenn man einen Menschen täuschen will, braucht man ihm nur das Bild eines Vorhangs vor Augen halten, das heißt das Bild von etwas, jenseits dessen er zu sehen verlangt.«

23 Der vielzitierte Satz, dass das Netz nicht vergesse (und dass das offenbar ein Problem ist), zeigt, dass man es hier (scheinbar) mit einem unkontrollierten mnemonischen Raum zu tun 
2. Ein zweiter Hinweis darauf, was man als eine medienhistorisch eigene Form des Blicks in der Gegenwart sehen könnte, kommt aus einer ganz anderen Richtung (und es sei betont, dass die beiden hier diskutierten Phänomene natürlich keineswegs die einzigen $\operatorname{sind}^{24}$ ): Lacan betont immerzu, dass das Subjekt im »Bild/Tableau « (Lacan 1987: 113) ist. Nur ist das "Bild «, in dem man sich befindet - und in dem auch der junge Lacan, bei den Fischern, »einen Fleck« (ebd.: 102) machte - ja kein zweidimensionales Bild. Es ist eine dreidimensionale Szene. Es ist ein dreidimensionales Bild.

Schon von daher ist es eigentlich merkwürdig, dass der Blick mit der Metapher der Fotografie beschrieben wird. Nochmal: »Daraus geht hervor, daß der Blick ein Instrument darstellt, mit dessen Hilfe das Licht sich verkörpert, und aus diesem Grund auch werde ich [...] photo-graphiert« (ebd.: 113). Wieso wird der Blick (the gaze), der doch so dem sgeometralen Phantasma des sehenden Subjekts entgegengesetzt sein soll (look), durch eine Technologie verkörpert, die - zumindest auf der Ebene der optischen Projektion - genau dieses geometrale, zentralperspektivische Schema wiederholt? Muss sie das, um das geometrale Phantasma des souveränen Subjekts zu stabilisieren? Oder liegt darin nicht ein Problem? Freilich könnte die Kamera des Blicks im Außen an jedem Punkt sein, ständig ihre Position wechseln und so virtuell eine Art omniskopische Kamera sein - eine Art sehr schneller Drohne. Man könnte aber auch die Frage aufwerfen, ob Silvermans (1996: 135) historisierende Perspektive - $[\mathrm{T}]$ he camera has been installed ever since the early nineteenth century as the primary trope through which the Western subject apprehends the gaze « - nicht auch die Möglichkeit zulässt, dass historisch neue und vielleicht sogar angemessenere primary tropes für den Blick auftauchen. Vielleicht ist die eher triviale Beobachtung, dass heutige Fotokameras (z.B. in Handys) digital und nicht mehr analog aufzeichnen gar nicht relevant. Vielleicht gibt es heute ganz andere Kameras.

Auf einigen wenigen Seiten des Seminar XI diskutiert Lacan Roger Caillois' (1984; 2007) einflussreiche Überlegungen zur Mimikry. Caillois hatte die in der Natur oft beobachtete Eigenschaft von Organismen, sich dem Hintergrund anzupassen, abweichend von der Darwin'schen Konzeption diskutiert. Lacan betont in Bezug auf Caillois zustimmend »Hier zeigt sich nun in der Tat, in welcher Dimension das Subjekt ins Tableau einrückt« (Lacan 1987: 106). Und wenn ein dreidimen-

hat, in der Subjekte Spuren hinterlassen, angeblickt, beurteilt, beschämt werden. Zu den Formen digitaler Bildzirkulation vgl. auch Prokić 2020.

24 Gerade Frauen, Angehörige der verschiedensten ssexuellen Minderheiten und People of Colour haben noch mit ganz anderen Formen des invektiven Blicks zu kämpfen. Diese Diskriminierungen stehen aber hier nicht im Zentrum, sondern die Frage nach neuen technischen Formen des Blicks. 
sionaler Körper (das Subjekt) in einen dreidimensionalen Hintergrund einrückt handelt es sich um ein dreidimensionales Bild und nicht um eine Fotografie, die ja eine zweidimensionale zentralperspektivische Projektion einer dreidimensionalen Szene ist (wobei die diesbezüglich eher vagen Formulierungen bei Lacan im Prinzip beide Möglichkeiten zuließen, wäre nicht so oft von Fotografie und Schirmen etc. die Rede). Caillois (1984: 23) bemerkt:

Morphological mimicry could then be, after the fashion of chromatic mimicry, an actual photography, but of the form and the relief, a photography on the level of the object and not on that of the image, a reproduction in three-dimensional space with solids and voids: sculpture-photography or better teleplasty, if one strips the word of any metapsychical content. ${ }^{25}$

Hier wird der Blick, also das Gesehenwerden-Können zwar immer noch in Metaphern der Fotografie verstanden - doch als eine »sculpture-photography«, als ein dreidimensionales Bild. Die Nähe dieser Formulierung zu der, schon im 19. Jahrhundert entstandenen, Technologie der Photoskulptur ist augenfällig (vgl. Schröter 2009: 92-126). Dies war ein Verfahren, bei welchem ein Subjekt in der Mitte einer Anordnung aus zahlreichen, kreisförmig angeordneten Kameras von eben diesen zugleich fotografiert wurde. Aus den Fotos, die das Subjekt von allen Seiten zeigten, konnte mit einigen Zwischenschritten eine Skulptur erstellt werden. Dies konnte selbstrepräsentative Zwecke erfüllen, es gab sogar die (aus naheliegenden Gründen) vorübergehende Praxis einer Art dreidimensionaler Visitenkarte (»bustes cartes«, vgl. Sorel 2000: 85). Die Details der sehr verschiedenen Verfahren sind hier nicht wichtig: Entscheidend ist nur, dass es eine Anordnung gibt, in der das Subjekt sehr buchstäblich von allen Seiten angeblickt wird, sodass keine zentralperspektivische Projektion der dreidimensionalen Szene aus einem Blickpunkt auf die zweidimensionale Fläche erfolgen muss. ${ }^{26}$

Was hat das nun mit dem invektiven Blick in der Gegenwart zu tun? Eine wichtige Technik der Globalisierung sind die an vielen Flughäfen installierten 3DKörperscanner, die ebenfalls eine Rundumabtastung erzeugen, um sicherzustellen, dass etwa keine Waffen in Flugzeuge geschmuggelt werden. Dieser durchdringende Rundum-Blick kann invektiv sein: In einem älteren Text heißt es:

Ceschlechtsorgane stehen auch aus einem weiteren Grund im Mittelpunkt der Diskussion über die Nacktscanner: Die Geräte bilden den unbekleideten Körper

25 Muhle (im Druck) hat diese Passage sehr genau in den Kontext von Caillois' Werk eingerückt und in dem Feld einer »Millieuästhetik« verortet. Siehe auch Eidelpes 2018: 67-84 zu einer detaillierten und sehr klaren Einordnung von Caillois' Position in den historischen Kontext. Jede einzelne Kamera bleibt (im Prinzip) zentralperspektivisch, aber die Vervielfältigung der Kamera führt dazu, dass nicht mehr eine räumliche Szene von einem Blickpunkt aus auf eine Bildfläche projiziert werden muss. 
detailreich ab. So kursieren bereits Geschichten von Angestellten, die Körperbilder eines Filmstars ausgedruckt und herumgezeigt haben sollen. An einem amerikanischen Flughafen sind Sicherheitsleute angeblich in eine Schlägerei geraten, nach einer abfälligen Bemerkung über den Penis des anderen. Immer wieder ist auch zu hören, attraktive Frauen würden besonders häufig >zufällig`für die Körperscans ausgewählt. (Schrader 2010).

Es geht nicht speziell um dieses Beispiel, auch sind bei modernen Systemen die Körper in der Darstellung stark schematisiert und frei von intimen Details. Solche Technologien der Sichtbarkeit zeigen, dass erneut der Blick des Anderen mich enthüllt, mich buchstäblich von meinem, auch schützenden Schirm trennt, und zum Objekt macht.

Doch läuft das an Flughäfen längst (meist) ohne Scham und ganz routinemäßig ab. Wie passt das in eine Blicktheorie nach Sartre und Lacan? Passt das nicht ohnehin besser in Konzepte einer panoptischen surveillance nach Foucault (1994)? Wozu dieser ganze subjekttheoretische Aufwand, wenn es einfach um Kontrolle und Disziplinierung geht? Das sind berechtigte Fragen - zumal es schon frühere Diskussionen darum gab (vgl. Copjec 1994). Man kann zunächst festhalten, dass das Dispositiv des 3D-Scanners (und der Photoskulptur), das Dispositiv des panoptischen Blicks, wie es Foucault beschreibt, umkehrt. Nicht mehr ist das Subjekt außen und wird dem Blick eines Zentrums ausgesetzt, sondern vielmehr ist das Subjekt im Zentrum und der Blick kommt »zentroramatisch« (Schmidt 2002: 186) von Außen - was Lacans (1987: 113) Bestimmung entspricht, dass der Blick »draußen« bzw. »im Außen« ist.

Abb. 2: Panorama vs. Zentrorama, nach Schmidt (2002).

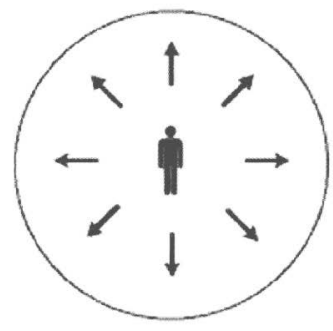

Panorama/Panopticon

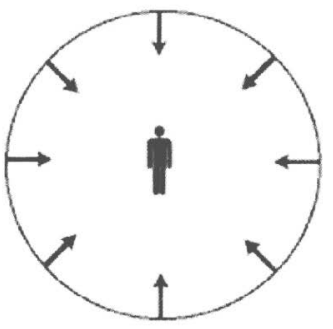

Zentrorama

Aber ist diese Umkehrung wirklich etwas anderes? Ist das Zentrum nicht einfach abstrakter als in dem von Foucault analysierten Gefängnisbau? Ist es nicht immer noch unsichtbar und beobachtet uns vielmehr durch die Netzwerke der Flughafeninstitutionen? Vielleicht macht es aber auch keinen Sinn, diese beiden 
Perspektiven gegeneinander auszuspielen - der technische Blick dient einmal der Überwachung und Kontrolle, ist andererseits in seinen verschiedensten Formen immer schon konstitutiv für zumindest einen bestimmten Typ von Subjektivität, wie er sich historisch herausgebildet hat.

3. Ähnliches könnte man, andeutungsweise, für zeitgenössische Technologien des (Self-)Trackings argumentieren. Da diese Diskussion anderswo ausführlich geführt wurde, sei nur kurz auf die einschlägige Literatur verwiesen (vgl. Duttweiler et al. 2016). Das Subjekt fühlt sich von einem vermessenden, quantifizierenden Blick angesehen, der es nicht mehr allein zu einem Objekt macht, das etwa einem Schirm aus hegemonialen Erscheinungsmustern folgt, sondern es wird $\mathrm{zu}$ einem Datensatz, der gerankt und verglichen wird - mit der sich wandelnden eigenen Performanz oder mit jener anderer. Auch hier kann es invektiv werden, etwa wenn die Leistung anderer als schwach bewertet wird. Allerdings stellt sich hier die Frage, ob die Metapher des Blicks (the gaze) wirklich noch zutreffend ist - vielleicht ist die Rolle, die der Blick in der Mitte des zwanzigsten Jahrhunderts spielte, dann eben doch ein Effekt der damaligen Dominanz visueller Medien wie der Fotografie und des Kinos (vgl. Kittler 1986: 249-251). ${ }^{27}$ Lacan schreibt eben, das Subjekt werde "photo-graphiert « und nicht: gescannt oder gar verdatet - und vielleicht ist selbst das vielzitierte Panoptikum nach Foucault noch zu sehr visuell, um den eher abstrakten und dann sekundär visualisierten Daten noch gerecht werden zu können. ${ }^{28}$ Vielleicht kommt nach dem invektiven Blick das invektive Ranking oder die invektive Statistik. ${ }^{29}$ Hier wird die oben schon erwähnte Invektivität gegenüber nicht-weißen Menschen noch einmal verschärft eine Rolle spielen, insofern der bias der Algorithmen schwarze Körper einerseits betont objektifiziert und zu Objekten eines Verdachts, andererseits (und in anderen Zusammenhängen) unsichtbar macht. ${ }^{30}$

Aber, selbst wenn die pan-optische Metapher nicht mehr greift, so bleibt doch, dass die Sichtbarkeit für andere (und sei es in der Form von peinlichen Daten und ihren Visualisierungen) konstitutiv für Subjektivität ist, ja sogar mehr ist als je

27 Vgl. Kittler 2002: 38: »Methodisch bleibt nur anzumerken, daß ich Lacans Begrifflichkeit als nützlichen Werkzeugkasten, nicht als unwandelbare Wahrheit benutzen werde - aus dem schlichten Grund, weil man sich im Lauf des Semesters wird fragen müssen, ob nicht Grundbegriffe aktueller Theorien, statt garantiert unabhängige und deshalb wahre Beobachtungsposten zu bilden, vielmehr eine direkte Folge der Medienexplosion unserer Epoche sind.«

28 Vgl. Surveillance and Society (2003) - das Heft versammelt eine Reihe von Beiträgen, die schon 2003 kritisch die Frage nach Foucaults Panoptizismus unter neuen medialen und sozialen Bedingungen stellen.

29 Vgl. hierzu Meyer 2019: Kapitel IV, der detailliert das Identifikationsregime der sozialen Medien unter Rückbezug Deleuze` und Guattaris Begriff der »abstrakten Maschine« analysiert. 
zuvor. Auch wenn die Kamera nicht mehr die richtige Metapher für den Blick sein mag - am Blick (the gaze) ändert sich dadurch nichts, nur die Formen, in denen er erscheint und mithin auch seine Invektivitäten haben sich verschoben.

\section{Literaturverzeichnis}

Barthes, Roland (1989): Die helle Kammer. Bemerkung zur Photographie [1980]. Frankfurt a.M.: Suhrkamp.

Baxandall, Michel (1988): Die Wirklichkeit der Bilder. Malerei und Erfahrung im Italien des 15. Jahrhunderts. Frankfurt a.M.: Suhrkamp.

Beil, Benjamin/Schröter, Jens (2011): »Die Parallelperspektive im digitalen Bild«. In: Zeitschrift für Medienwissenschaft. Heft 04: Menschen \&Andere. Jg. 3, Nr. 1, S. 127-138. DOI: 10.1524/zfmw.2011.0011.

Blümle, Claudia/von der Heiden, Anne (Hgg.) (2005): Blickzähmung und Augentäuschung. Zu Jacques Lacans Bildtheorie. Zürich: Diaphanes.

Bryson, Norman (1998): »The Gaze in the Expanded Field«. In: Foster, Hal (Hg.): Vision and Visuality. Seattle: Bay Press, S. 87-113.

Butler, Judith (1999): Gender Trouble. Feminism and the Subversion of Identity. New York/London: Routledge.

Caillois, Roger (1984): »Mimicry and Legendary Psychasthenia«. In: October, Nr. 31, S. 16-32.

Caillois, Roger (2007): Méduse \&Cie. Die Gottesanbeterin. Mimese und legendäre Psychastenie. Berlin: Brinkmann und Bose.

Copjec, Joan (1994): Read My Desire. Lacan against the Historicists. Cambridge/MA; London: MIT Press.

Därmann, Iris (2000): »Unter dem Blick bildlicher Medien«. In: Pöltner, Günther (Hg.): Phänomenologie der Kunst. Frankfurt a.M.: Peter Lang, S. 13-42.

Duttweiler, Stephanie/Gugutzer, Robert/Passoth, Jan-Hendrik et al. (Hgg.) (2016): Leben nach Zahlen. Self-Tracking als Optimierungsprojekt? Bielefeld: transcript. DOI: $10.14361 / 9783839431368$.

Eidelpes, Rosa (2018): Entgrenzung der Mimesis. Georges Bataille-Roger Caillois-Michel Leiris. Berlin: Kadmos.

Fanon, Frantz (1952): Peau noire, Masques blancs. Paris: Seuil.

Fanon, Frantz (2008): Black Skin, White Masks. London: Pluto Press.

Foucault, Michel (1994): Überwachen und Strafen [1975]. Frankfurt a.M.: Suhrkamp. Gondek, Hans-Dieter (1997): »Der Blick - zwischen Sartre und Lacan. Ein Kommentar zum VII. Kapitel des Seminars XI«. In: Riss. Zeitschrift für Psychoanalyse, Jg. 12, Nr. 37/38, S. 173-194. 
Görling, Reinhold (2017): »Szenische Verfasstheit der Subjektivität, Medienökologie der Psyche«. In: Zeitschrift für Medienwissenschaft. Heft 17: Psychische Apparate, Jg. 9, Nr. 2, S. 41-53. DOI: 10.14361/zfmw-2017-0206.

Iskin, Ruth E. (1997): »In the Light of Images and the Shadow of Technology. Lacan, Photography and Subjectivity«. In: Discourse, Jg. 19, Nr. 3, S. 43-66.

Iversen, Margaret (2002): »Was ist eine Fotografie? « In: Wolf, Herta (Hg.): Paradigma Fotografie. Fotokritik am Ende des fotografischen Zeitalters, Bd. 1. Frankfurt a.M.: Suhrkamp, S. 92-114.

Jay, Martin (1993): Downcast Eyes. The Denigration of Vision in Twentieth Century French Thought. Berkeley u.a.: University of California Press.

Kittler, Friedrich (1986): Grammophon Film Typewriter. Berlin: Brinkmann und Bose. Kittler, Friedrich (2002): Optische Medien. Berliner Vorlesung 1999. Berlin: Merve.

Lacan, Jacques (1986): »Von dem, was uns vorausging«. In: Ders.: Schriften III. Weinheim/Berlin: Quadriga, S. 7-14.

Lacan, Jacques (1987): Die vier Grundbegriffe der Psychoanalyse. Das Seminar Buch XI. Weinheim/Berlin: Quadriga.

Merleau-Ponty, Maurice (1986): Das Sichtbare und das Unsichtbare. München: Fink.

Meyer, Roland (2019): Operative Portraits. Eine Bildgeschichte der Identifizierbarkeit von Lavater bis Facebook. Konstanz: Konstanz University Press.

Muhle, Maria (im Druck): ")Eine Skulptur-Photographie oder besser eine Teleplastik« - Mimesen zwischen Natur und Kultur bei Caillois«. In: Balke, Friedrich/Linseisen, Elisa (Hgg): Mimesis Expanded, München: Fink (im Druck).

Noble, Safiya Umoja (2018): Algorithms of Oppression. How Search Engines reinforce Racism. New York: New York University Press.

Owens, Craig (2003): »Posieren«. In: Wolf, Herta (Hg.): Diskurse der Fotografie. Fotokritik am Ende des fotografischen Zeitalters, Bd. 2. Frankfurt a.M.: Suhrkamp, S. $92-114$.

Prokić, Tanja (2020): »Post, Like, Share, Submit. Visual Control and the Digital Image (13 Theses)«. In: Control Societies I: Media, Culture, Technology. Special Issue of Coils of the Serpent: Journal for the Study of Contemporary Power, Jg. 5, Nr. 1, S. 145-152.

Sartre, Jean-Paul (1991): Das Sein und das Nichts. Versuch einer phänomenologischen Ontologie [1943]. In: Ders.: Sartre. Gesammelte Werke in Einzelausgaben. Philosophische Schriften, Bd. 3. Hg. v. Traugott König. Hamburg: Rowohlt.

Schmidt, Gunnar (2002): »Zeit des Ereignisses - Zeit der Geschichte. Am Beispiel der Multiperspektivität«. In: Chi, Immanuel/Düchting, Susanne/Schröter, Jens (Hgg.): ephemer_temporär_provisorisch. Essen: Klartext, S. 175-196.

Schrader, Christopher (2010): »Entwürdigend und gefährlich«. In: Süddeutsche Zeitung. https://www.sueddeutsche.de/wissen/nacktscanner-entwuerdigendund-gefaehrlich-1.1024256 [Zuletzt eingesehehen 14.7.2021]. 
Schröter, Jens (2003): »Virtuelle Kamera. Zum Fortbestand fotografischer Medien in computergenerierten Bildern«. In: Fotogeschichte, Jg. 23, Nr. 88, S. 3-16.

Schröter, Jens (2004): »Das Ende der Welt. Analoge vs. digitale Bilder - mehr und weniger >Realität‘?«. In: Ders./Böhnke, Alexander (Hgg.): Analog/Digital - Opposition oder Kontinuum? Zur Theorie und Geschichte einer Unterscheidung. Bielefeld: transcript, S. 335-354. DOI: 10.14361/9783839402542-017.

Schröter, Jens (2009):3D. Zur Geschichte, Theorie und Medienästhetik des technisch-transplanen Bildes. München: Fink.

Schröter, Jens (2016): »Sich ausweisen«. In: Becker, Ilka/Lockemann, Bettina/Köhler, Astrid et al. (Hgg.): Fotografisches Handeln. Das fotografische Dispositiv, Bd 1. Marburg: Jonas, S. 226-239.

Schröter, Jens (2019): Löschbarkeit und Negation. In: Nowak, Lars (Hg.): Bild und Negativität. Würzburg: Königshausen \& Neumann, S. 143-154.

Silverman, Kaja (1996): The Threshold of the Visible World. New York: Routledge.

Sorel, Philippe (2000): »Photosculpture: the Fortunes of a Sculptural Process Based on Photography«. In: Reynaud, Françoise/Tambrun, Catherine/Timby, Kim (Hgg.): Paris in 3D. London: Booth-Clibborn Editions, S. 81-90.

Surveillance and Society (2003): Foucault and Panopticism Revisited. https://ojs.lib rary.queensu.ca/index.php/surveillance-and-society/issue/view/Foucault [Zuletzt eingesehehen 14.7.2021].

Sycha, Lisa (2020): Billie Eilish: Beschimpfungen wegen Bikini-Fotos im Urlaub. In: Rolling Stone. https://www.rollingstone.de/billie-eilish-beschimpfungen-wegen-b ikini-fotos-im-urlaub-1968963/ [Zuletzt eingesehehen 14.7.2021].

Tifentale, Alisa/Manovich, Lev (2015): »Selfiecity: Exploring Photography and SelfFashioning in Social Media«. In: Berry, David M./Dieter, Michael (Hgg.): Postdigital Aesthetics: Art, Computation and Design. Basingstoke (Hampshire): Palgrave Macmillan, S. 109-122. DOI: 10.1057/9781137437204_9.

Tomšič, Samo (2015): The Capitalist Unconscious. Marx and Lacan. London: Verso. 
